\title{
Oxygen Dissociation by Concerted Action of Di-Iron Centers in Metal-Organic Coordination Networks at Surfaces: Modeling Non-Heme Iron Enzymes
}

\author{
Stefano Fabris, ${ }_{\S}^{*,+, \neq}$ Sebastian Stepanow, ${ }^{*, \S}$ Nian Lin, ${ }_{\S,}^{*,, \|}$ Pietro Gambardella, ${ }^{\perp}$ Alexandre Dmitriev, ${ }^{\S, \nabla}$ \\ Jan Honolka, ${ }^{\S}$ Stefano Baroni, ${ }^{+, *}$ and Klaus Kern ${ }^{\S, \#}$ \\ ${ }^{+}$CNR-IOM DEMOCRITOS, Theory@Elettra Group, Istituto Officina dei Materiali, c/o Sincrotrone Trieste-SS14, Km 163, \\ 5 Basovizza, I-34012 Trieste, Italy \\ ${ }^{\ddagger}$ SISSA Scuola Internazionale Superiore di Studi Avanzati, Via Bonomea 265, 34136 Trieste, Italy \\ ${ }^{\S}$ Max-Planck Institute for Solid State Research, Heisenbergstrasse 1, 70569 Stuttgart, Germany \\ "Department of Physics, The Hong Kong University of Science and Technology, Clear Water Bay, Hong Kong, China \\ ${ }^{\perp}$ ICREA and Catalan Institute of Nanotechnology (ICN-CIN2), E-08193 Barcelona, Spain \\ ${ }^{*}$ Institut de Physique de la Matière Condensée, Ecole Polytechnique Fédérale de Lausanne, 1015 Lausanne, Switzerland
}

Supporting Information

ABSTRACT: The high chemical reactivity of unsaturated metal sites is a key factor for the development of novel devices with applications in sensor engineering and catalysis. It is also central in the research for sustainable energy concepts, e.g., the efficient production and conversion of chemical fuels. Here, we study the process of oxygen dissociation by a surface-supported metalorganic network that displays close structural and functional analogies with the cofactors of non-heme enzymes. We synthesize a two-dimensional array of chemically active di-iron sites on a $\mathrm{Cu}(001)$ surface where molecular oxygen readily dissociates at

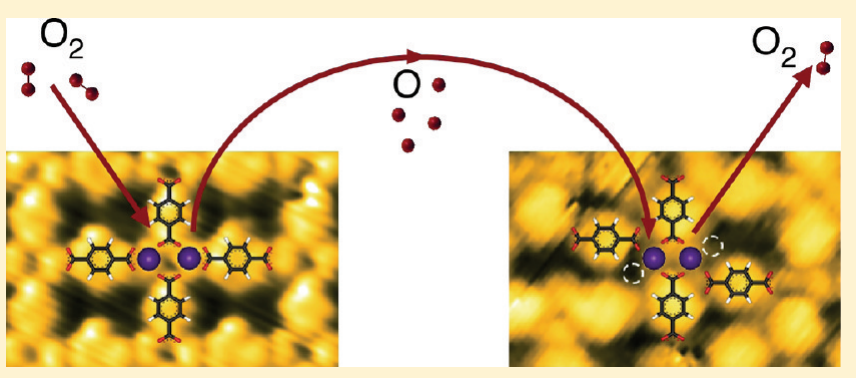
room temperature. We provide an atomic-level structural and electronic characterization before and after reaction by combining scanning tunneling microscopy, X-ray absorption spectroscopy, and density functional theory. The latter identifies a novel mechanism for $\mathrm{O}_{2}$ dissociation controlled by the cooperative catalytic action of two $\mathrm{Fe}^{2+}$ ions. The high structural flexibility of the organic ligands, the mobility of the metal centers, and the hydrogen bonding formation are shown to be essential for the functionality of these active centers allowing to mimick biologically relevant reactions in a confined environment.

KEYWORDS: Heterogeneous catalysis, synthetic enzymes, oxygen activation, STM, DFT

\begin{abstract}
$\mathrm{O}$ pen metal sites in nanostructured coordination compounds have gained extensive attention due to their potential applications in catalysis, gas adsorption, and molecular recognition. ${ }^{1-3}$ Moreover, metal centers are found to be effective catalysts for the efficient conversion and production of chemical fuels for the sustainable provision of energy. ${ }^{4-6}$ Coordinatively unsaturated metal sites play a key role in many organic and inorganic transformations as well as in several biological processes. ${ }^{7,8}$ For instance, dinuclear metal centers are a common structural feature in the cofactor of many metalloproteins controlling respiration or metabolism as well as in biomimetic catalysts for renewable energy applications, such as artificial photosynthesis. ${ }^{9-12}$ Several of these fundamental units are based on $\mathrm{Fe}$ ions that catalyze the dissociation of molecular oxygen. ${ }^{13}$ A remarkable functional versatility is displayed by carboxylate-bridged Fe ions, which is also a typical structural motif in non-heme enzymes. They allow for the participation of oxygen into a large variety of chemical reactions controlling important processes, such as the oxidation
\end{abstract}

of methane to methanol, ${ }^{14}$ the conversion of nucleotides to deoxynucleotides, ${ }^{15}$ or the conversion of alkanes to alkenes. ${ }^{16}$ The structural characterization of the carboxylate-bridged iron units present in most of these metalloproteins has been the subject of extensive work, ${ }^{17-21}$ and even more efforts have been dedicated to the understanding of their reaction mechanisms, a formidable challenge due to the complexity of the biological environment. $^{20,21}$

Fundamental insight into the active site functionality has been achieved by focusing on simpler synthetic systems that model elementary sites of biological units. ${ }^{22}$ One successful class of such model systems is based on sterically hindered carboxylic acids capable to stabilize di-iron(II) complexes. These are shown to react in solution with $\mathrm{O}_{2}$ and to display characteristic ligand

Received: September 12, 2011

Revised: October 14, 2011

Published: October 19, 2011 
displacement and substitution during the reaction. These models mimic the chemical and structural environments present in specific regions of metalloproteins and have contributed to reveal important aspects controlling their functions. ${ }^{22}$ In addition, the study of model synthetic structures can have implications in other fields of nanotechnology, since they can display catalytic functions regulated by completely new reaction mechanisms.

An alternative approach to steric hindrance relies on supramolecular engineering. This strategy can lead to the formation of highly ordered 2D metal-organic coordination networks (MOCNs) on atomically flat metal surfaces. ${ }^{23,24}$ The resulting nanostructures provide a unique opportunity for exploring the reactivity of coordinatively unsaturated metal centers under wellcontrolled chemical and physical conditions. ${ }^{25-28}$ In this work, we investigate the structure and reactivity of two-dimensional (2D) MOCNs comprising of carboxylate-bridged mono- and diiron sites coordinated to terephthalic acid (1,4-benzenedicarboxylic acid, TPA). We show that the active sites exposed by these MOCNs display structural and functional similarities with those of biological and of model sterically hindered di-iron complexes. We propose that this synthetic nanostructure dissociates molecular $\mathrm{O}_{2}$ with a reaction mechanism that exploits a cooperative catalytic action of the two Fe centers in the active site.

The local structure was studied with in situ scanning tunneling microscopy (STM) sequentially between defined oxygen exposures or directly in an oxygen atmosphere. The MOCNs were further characterized before and after $\mathrm{O}_{2}$ exposure with X-ray absorption spectroscopy (XAS) at the $\mathrm{Fe} L_{2,3}$-edge, while their atomic structure, thermodynamics, and reactivity were studied with density functional theory (DFT) calculations. Details of the sample preparation procedure and the STM and XAS measurements as well as of the DFT calculations ${ }^{29}$ are reported in the Supporting Information.

Figure 1a,e shows high-resolution STM topographs (obtained in situ after the synthesis in ultrahigh vacuum conditions) of the two types of metal-coordination networks that spontaneously self-assemble from TPA molecules and iron atoms on a $\mathrm{Cu}(100)$ surface. Depending on the employed metal-to-molecule concentration ratio, controlled formation of $2 \mathrm{D}$ MOCN consisting of either di-iron or monoiron (Figure 1a,e, respectively) coordination centers can be achieved. The structural models are superimposed to the images in Figure 1a and e. The Fe atoms, represented as purple spheres, coordinate to four adjacent oxygen atoms of the carboxylate moieties of the TPA ligands resulting in a square-planar coordination geometry. ${ }^{25}$

Figure $1 \mathrm{a}-\mathrm{d}$ displays representative STM images acquired after defined oxygen dosage and reveals the structural modifications of di-iron MOCNs as a function of $\mathrm{O}_{2}$ exposure. This sequence of images (see also Supporting Information) shows that the highly ordered and periodic initial di-iron MOCN structure (Figure 1a) is strongly modified upon exposure to molecular oxygen at room temperature $(300 \mathrm{~K})$ and is dismantled step-bystep with increasing oxygen dosage (Figure $1 \mathrm{~b}-\mathrm{d}$ ). At a dosage of $27 \mathrm{~L}$ (partial $\mathrm{O}_{2}$ pressure of $3 \times 10^{-8} \mathrm{mbar}$ ), the time scale of the reaction is of the order of minutes. As discussed in the following, we ascribe the disordering and dismantling of the network structure to the dissociation of the molecular oxygen upon selective adsorption at the di-iron units. XAS spectroscopy provides complementary evidence that the di-iron units are chemically modified as a result of their interaction with oxygen.

The active response of di-iron centers contrasts with the behavior of the monoiron centers, which selectively bind oxygen
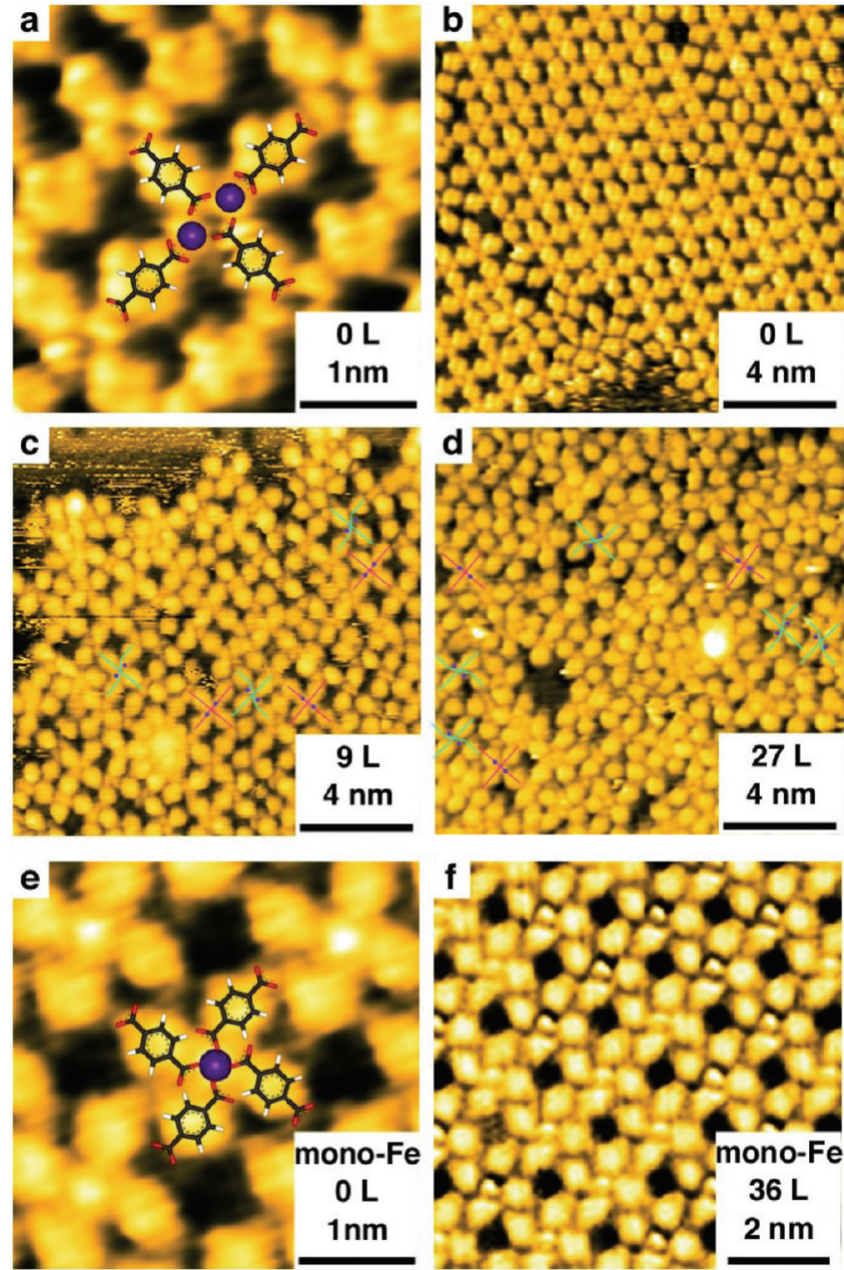

Figure 1. STM images of the MOCNs exposed to oxygen. Reaction of di-iron $(\mathrm{a}-\mathrm{d})$ and monoiron $(\mathrm{e}, \mathrm{f})$ MOCNs with molecular oxygen as a function of $\mathrm{O}_{2}$ dosage (in Langmuir, L). Purple circles represent $\mathrm{Fe}$ centers. In panels $\mathrm{c}$ and $\mathrm{d}$, selected pristine and $\mathrm{O}$-modified units are highlighted by purple and cyan lines, respectively. STM imaging conditions: $I=0.1 \mathrm{nA}, U=0.3-0.5 \mathrm{~V}$, and $T=300 \mathrm{~K}$.

molecules at the iron sites but do not undergo a structural transition even when exposed to larger doses of $\mathrm{O}_{2}$ (Figure 1e, f). ${ }^{30}$ The iron centers with adsorbed oxygen molecules appear with a different contrast in the STM image in Figure 1f. As discussed later, such a drastically different behavior of mono- and di-iron centers in a similar oxygen background illustrates the extreme sensitivity of the oxygen dissociation reaction activated at the coordination centers with respect to their atomic structure.

Figure 2a displays the high-resolution STM image of the diiron MOCN before exposure to molecular oxygen (initial state) together with the corresponding equilibrium structure predicted by the DFT calculations (Figure 2c). The comparison between the simulated (Figure 2e, inset in Figure 2a) and the experimental STM images allows us to associate the large diamond-shaped objects to the aromatic ligands and the small circles to the iron centers, in agreement with previous analysis. ${ }^{31}$ The molecule-tosurface interaction aligns all ligands with their aromatic rings parallel to the metal surface. Each iron is coordinated by a chelating bidentate carboxylate along the $\mathrm{Fe}-\mathrm{Fe}$ axes (horizontal ligands in Figure 2) and by two bridging bis-bidentate carboxylates (vertical ligands in Figure 2). This results in a distorted 

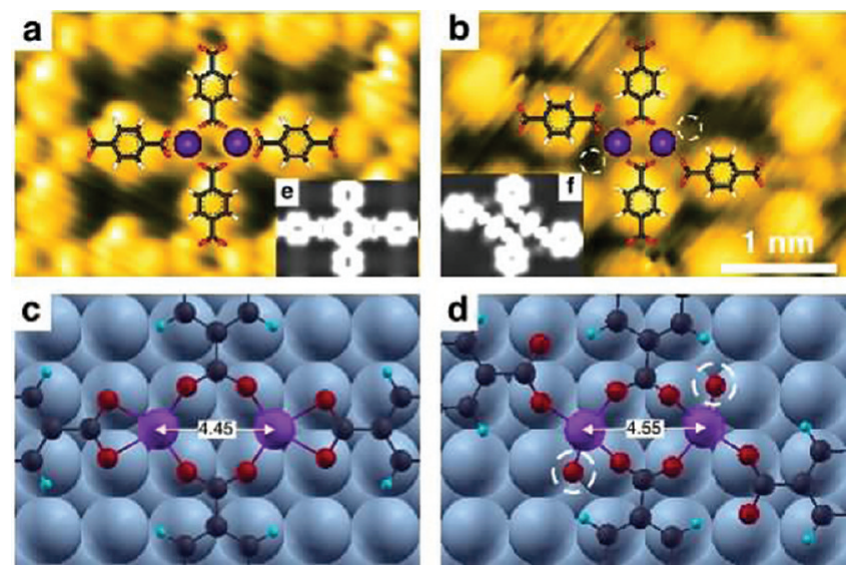

Figure 2. High-resolution STM topograph of the di-iron MOCN before oxygen exposure (a) and oxygen modified phase after $27 \mathrm{~L}$ of $\mathrm{O}_{2}$ exposure (b), corresponding equilibrium geometries ( $\mathrm{c}$ and $\mathrm{d}$ ) and simulated STM images predicted by the DFT calculations (insets, e and $\mathrm{f})$. Color code: carbon in gray, $\mathrm{Fe}$ in violet, oxygen in red, hydrogen in cyan, and $\mathrm{Cu}$ in light blue; $\mathrm{T}=300 \mathrm{~K}$.

square-planar Fe coordination by oxygen atoms, leading to the calculated $\mathrm{Fe}-\mathrm{O}$ bond lengths of 2.02 and $2.25 \AA$ for the bridging and chelating ligands, respectively. The calculated distance between the two Fe centers is $4.45 \AA$, while their elevation over the $\mathrm{Cu}(100)$ surface is $2.06 \AA$, much higher than Fe adatoms adsorbed to the same surface (1.51 $\mathrm{A})$. We conclude that the diiron unit is pulled outward from the metal surface by the strong $\mathrm{Fe}$-carboxylate bonding. Our electronic structure calculations predict that the di-iron unit can be identified as pair of high-spin $\mathrm{Fe}^{2+}$ ions.

Exposure to molecular oxygen gradually destroys the longrange order of the pristine phase, as clearly shown by the STM images displayed in Figure 1c,d, corresponding to 9 and $27 \mathrm{~L}$ of oxygen, respectively. In these STM topographs, some of the diiron units in the pristine phase described above are still present: The metal centers are highlighted by purple circles and their TPA ligands by magenta cross lines. The interaction with $\mathrm{O}_{2}$ induces drastic modifications, and after $27 \mathrm{~L}$ very few units of the pristine phase are still present, leading to a disordered assembly of TPA molecules on the surface in which several different local arrangements can be identified. At the initial steps of oxygen adsorption, a characteristic structure is observed together with the unaffected pristine phase, and it appears more and more often as a function of the increasing oxygen exposure. Eventually, at sufficiently high-oxygen exposure disorder prevails mainly driven by the thermal-induced diffusion of the adsorbates at room temperature. Some of these characteristic O-modified di-iron units are marked by cyan lines in Figure 1c,d. In this structure, the metal centers (purple circles) maintain their di-iron configuration, but the two axially binding TPA ligands are displaced with respect to their position in the pristine phase. This structure, an intermediate for the more disordered molecular assemblies present in the STM topographs, provides insight into the reaction path between the carboxylate-bridged di-iron unit and molecular $\mathrm{O}_{2}$.

A high-resolution STM image of this characteristic di-iron MOCN structure modified by oxygen exposure (27 L of oxygen) is shown in Figure $2 \mathrm{~b}$ (final state). To a first approximation, the iron centers and the two bridging (vertical) ligands preserve the same position as in the initial state. Also the two bridging carboxylate groups retain the bis-bidentate bonding to the dinuclear irons, whereas the two horizontal ligands undergo a shift in the surface plane by $\sim 2 \AA$. In such geometry, they cannot sustain anymore the chelating bidentate bonding to the $\mathrm{Fe}$ center. Instead they form monodentate bonding with the iron atoms. The lowest energy structure predicted by the DFT analysis is compatible with this ligand shift and is shown in Figure 2d. We note that the ligand shift leaves the Fe centers strongly undercoordinated (three-fold) and that a very similar shift is also observed in the synthetic, sterically hindered carboxylate-di-iron units after reacting in solution with $\mathrm{O}_{2}{ }^{22,32,33}$ In that case, the $\mathrm{O}$ atoms resulting from the $\mathrm{O}_{2}$ dissociation become the fourth ligand thus recovering the four-fold coordination of each Fe center and therefore being the driving force for the carboxylate shift.

Our calculations show that the structure imaged by STM is indeed compatible with the presence of two additional $\mathrm{O}$ atoms (marked in Figure $2 \mathrm{~b}, \mathrm{~d}$ by dashed circles and denoted as $\mathrm{O}^{*}$ hereafter) in the di-iron unit. They result from $\mathrm{O}_{2}$ dissociation and bind to the Fe centers. The simulated STM image for the lowest energy structure (Figure 2f, inset in Figure 2b) is in good agreement with the experimental findings. We also note that the adsorption of molecular $\mathrm{O}_{2}$ as the fourth ligand to the Fe centers would result in strong features in the STM image, that are not present in Figure 2b (Supporting Information, Figure S3). With respect to the pristine phase, the calculated height of the $\mathrm{Fe}$ centers over the $\mathrm{Cu}$ surface and the distance between the $\mathrm{Fe}$ centers increase by 0.14 (to 2.20 ) and $0.1 \AA$ (to $4.55 \AA$ ), respectively. The bond lengths between the metal centers and the $\mathrm{O}$ atoms of the carboxylate groups are weakly affected by the reaction (2.02 $\AA$ in the pristine, $1.93-2.12 \AA$ in the O-modified structures), while the fourth $\mathrm{Fe}-\mathrm{O}^{*}$ bond length is shorter, $1.80 \AA$. The $\mathrm{O}^{*}$ atoms are not in the same plane of the organic ligands but are $0.88 \AA$ below them, in contact with the $\mathrm{Cu}$ surface. This geometrical argument explains the lack of STM signal in the region of the $\mathrm{O}^{*}$ atom displayed by both the experimental and simulated images (see dashed circles in Figure $2 b, d$ ). In summary, we correlate the ligand shift from bidentate to monodentate with the presence of $\mathrm{O}^{*}$ adatoms underneath the MOCN plane.

In order to obtain information on the chemical state of the iron atoms embedded in the metal-organic network we acquired XAS spectra at the Fe L-edge. Figure 3 shows the Fe $L$-edge spectra of the pristine di-iron network phase (denoted as $\left.\mathrm{Fe}_{2}(\mathrm{TPA})_{4}\right)$ and the modified structure $\left(\mathrm{Ox}-\mathrm{Fe}_{2}(\mathrm{TPA})_{4}\right)$. As a reference, we also measured the $L$-edge spectra of Fe deposited on the clean $\mathrm{Cu}(100)$ surface at room temperature (denoted as $\mathrm{Fe} / \mathrm{Cu}(100)$ in Figure 3) with an equivalent Fe concentration. The comparison of the XAS line shapes in Figure 3 reveals pronounced differences. $\mathrm{Fe} / \mathrm{Cu}(100)$ exhibits broad $L_{3}$ and $L_{2}$ peaks typical of a metal, whereas the metal-coordination compounds show a pronounced narrowing of the spectral features with the appearance of two shoulders at 706.5 and $711 \mathrm{eV}$ at the $L_{3}$ edge and a double peak structure at the $L_{2}$ edge. Such atomiclike multiplet features are typical of Fe ions in bulk metalorganic compounds ${ }^{34-36}$ and demonstrate the formation of coordination bonds between the Fe centers and the carboxylate ligands accompanied by localization of the Fe d-orbitals and partial decoupling of $\mathrm{Fe}$ from the $\mathrm{Cu}$ substrate. A comparison of the XAS line shape of the di-iron network with the monoiron phase reported in ref 30 reveals nearly identical XAS spectra, which signifies that the Fe centers are embedded in similar ligand environments and have predominantly $3 \mathrm{~d}^{6}$ character. 


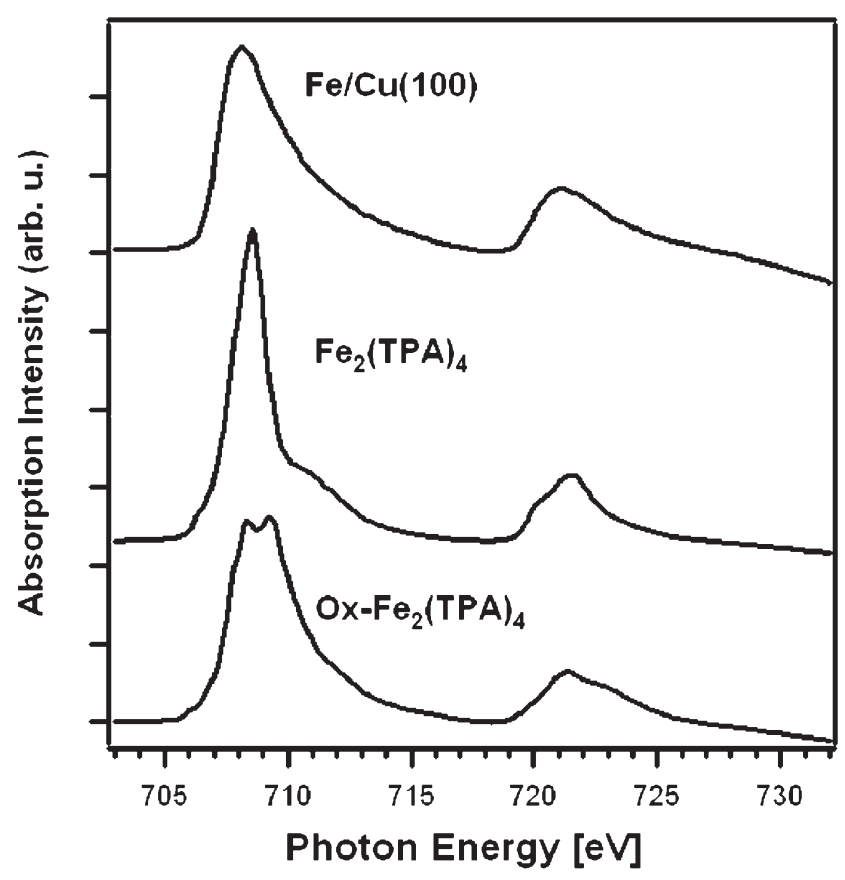

Figure 3. Fe $L_{3,2}$-edge XAS of the dinuclear Fe units before and after (middle and bottom spectrums, respectively) $100 \mathrm{~L}$ oxygen exposure. The upper curve shows the XAS of an equivalent amount of Fe deposited on the clean $\mathrm{Cu}(100)$ surface at room temperature. The spectra have been shifted vertically for clarity.

Oxygen dosing significantly changes the XAS features, revealing modifications of the chemical state of the Fe centers. In the $L_{3}$ region two peaks at 708.4 and $709.3 \mathrm{eV}$ are clearly identified. The $L_{2}$-edge also differs from the pristine network having now increased intensity at the high-energy side of the peak. The overall shift of spectral intensity to higher energy indicates charge depletion at the Fe centers, consistent with the fact that the spectrum of the oxygen-modified phase resembles that of $\mathrm{Fe}$ systems with higher oxidation states, i.e., $\mathrm{Fe}$ (III) or a mixture of $\mathrm{Fe}(\mathrm{II})$ and $\mathrm{Fe}$ (III) ions. ${ }^{34-36}$ Due to the inhomogeneity of the ligand environment of the Fe centers in the oxygen-modified phase, it is not practical to analyze the XAS spectrum with atomic multiplet calculations. However, the XAS line shape of the oxygenated di-iron network differs from the stable oxygenated monoiron phase. $^{30}$ Taking into account the disorder in the oxygen modified di-iron phase and hence altered ligand arrangements, the observed spectral changes are consistent with both oxidation and ligand field modifications. In addition, also a mixed $\mathrm{Fe}(\mathrm{II})$ and $\mathrm{Fe}$ (III) scenario is conceivable and accounts for the overall broadening of the XAS spectrum.

The calculated Löwdin charges of the Fe centers before and after reaction with $\mathrm{O}_{2}$ support the interpretation of the XAS spectra described above (Supporting Information, Table S4). ${ }^{37}$ Indeed, the formation of the $\mathrm{Fe}-\mathrm{O}^{*}$ bond decreases the $\mathrm{Fe}$ net charge by $\sim 0.16$ e (i.e., the $\mathrm{Fe}$ ions become more positive), while it increases the $\mathrm{O}^{*}$ charge by 0.44 e (the $\mathrm{O}^{*}$ becoming more negative) with respect to the corresponding values in the pristine structure. The calculated charges of the $\mathrm{O}$ atoms of the carboxylate groups are instead unmodified (changes smaller than $0.01 \mathrm{e}$ ) by the reaction. This charge disproportionation of $\sim 0.16 \mathrm{e}$ between the Fe centers in the two structures is compatible with the one calculated in related systems (in the range between 0.15 and 0.3 e in $\mathrm{Fe}$-phthalocyanine $\mathrm{e}^{38}$ or $\mathrm{Fe}_{3} \mathrm{O}_{4}$ spinel $)^{39}$ and attributed to the difference between $\mathrm{Fe}$ (II) and $\mathrm{Fe}$ (III) ions.

In principle, there are two possible reaction sites of the pristine phase that could catalyze $\mathrm{O}_{2}$ dissociation, either the undercoordinated metal centers of the MOCNs or the $\mathrm{Cu}$ surface exposed in the MOCN cavities. Between these, our DFT calculations predict that the relevant mechanism is governed by the reactivity of the dinuclear Fe centers rather than by the oxidation of the exposed $\mathrm{Cu}$ substrate. This conclusion is based on the activation energies of the rate-limiting steps in the two competing mechanisms, which are described in the following. Moreover the nonreactivity of the mono-Fe phase excludes the reaction channel via the $\mathrm{Cu}$ surface. The monoiron structure exhibits small cavities exposing one $\mathrm{Cu}$ hollow site for the adsorption of molecular oxygen. Although the presence of organic ligands could significantly alter the dissociation barrier and thus reduce the reaction rate with the network, the network was found to remain stable upon exposure to oxygen on a much larger time scale (minimum $12 \mathrm{~h}$, time of measurements). This is corroborated by the stability toward oxygen adsorption of a related monoiron structure denoted as double-row phase in ref 25 . The local coordination of the Fe centers as well as the cavity sizes are very similar to the di-iron structure, however the Fe atoms are embedded as single coordination centers (see Supporting Information for more information). This demonstrates that while oxygen binds on top of the Fe atoms, no further reaction takes place at the monoiron structures.

The dissociation of molecular oxygen on the coordinatively unsaturated $\mathrm{Fe}$ centers comprises of three fundamental steps: (i) the adsorption of molecular $\mathrm{O}_{2}$ to the metal centers of the diiron unit, (ii) the dissociation of the adsorbed molecules catalyzed by the metal centers, and (iii) the ligand shift leading to the oxygen-modified structure characterized above. The energetics of the adsorption and dissociation steps as well as the atomistic structure of the relevant intermediates resulting from the DFT calculations are reported in Figure 4.

The numerical simulations predict that a molecule of $\mathrm{O}_{2}$ adsorbs on one of the $\mathrm{Fe}^{2+}$ centers in the di-iron unit with a $\mathrm{Fe}-\mathrm{O}_{2}$ binding energy of $0.79 \mathrm{eV}$ (Figure 4). The large $\mathrm{Fe}-\mathrm{Fe}$ distance in the pristine phase $(4.45 \AA)$ prevents the direct formation of a $\mathrm{Fe}-\mathrm{O}-\mathrm{O}-\mathrm{Fe}$ bridging intermediate (Figure 4a2), which turns out to be unstable. Instead, another $\mathrm{O}_{2}$ molecule can be accommodated on the second metal center of the di-iron unit, with a calculated binding energy of $0.57 \mathrm{eV}$. This leads to a very stable $\mathrm{O}_{2}-\mathrm{Fe}-\mathrm{Fe}-\mathrm{O}_{2}$ configuration, which is the initial state for $\mathrm{O}_{2}$ dissociation (Figure 4a1,a3).

The presence of multiple adsorbates on the di-iron unit points to a complex reaction mechanism involving two $\mathrm{O}_{2}$ molecules and two metal centers. Our characterization of the final state $(\mathrm{O}-$ modified phase) predicts the presence of two $\mathrm{O}^{*}$ atoms that, in this reaction path, originate from the dissociation of the molecular adsorbates. The minimum energy reaction path for the dissociation processes obtained from the calculations is described in Figure 4. The main findings are that: (i) the dissociation of the two adsorbed $\mathrm{O}_{2}$ molecules takes place in sequence, and (ii) the second dissociation reaction takes advantage of the presence of a dissociated $\mathrm{O}_{2}$ molecule on the other Fe center of the same di-iron unit. The first dissociation is the rate-limiting step (Figure 4a4), with a calculated activation energy of $0.74 \mathrm{eV}$, while the dissociation of the second molecule requires only $0.25 \mathrm{eV}$ (Figure 4a5). The reason for this lower value lies in the specific dinuclear structure of the reaction center. Along the minimum 

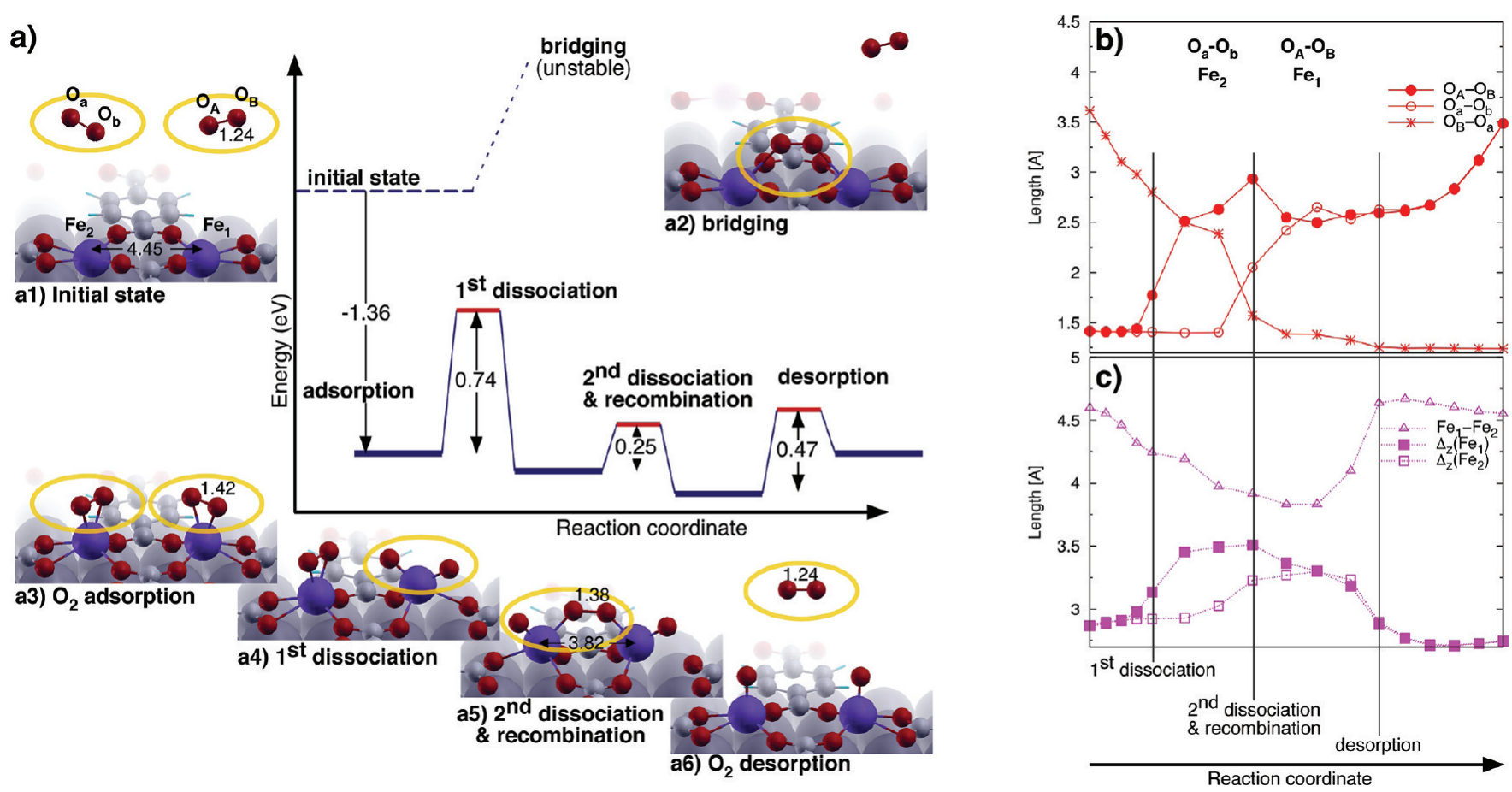

Figure 4. (a) Reaction mechanism for $\mathrm{O}_{2}$ dissociation predicted by the DFT calculations. The relative energies of the reaction intermediates (a1-a6) as well as the activation barriers are expressed in $\mathrm{eV} .(\mathrm{b}, \mathrm{c}) \mathrm{O}-\mathrm{O}, \mathrm{Fe}-\mathrm{Fe}$ bond lengths and elevation of the Fe ions with respect to the metal surface $\left(\Delta_{z}\right)$ along the reaction path (in $\AA$ ). $\mathrm{O}_{\mathrm{A}}-\mathrm{O}_{\mathrm{B}}$ and $\mathrm{O}_{\mathrm{a}}-\mathrm{O}_{\mathrm{b}}$ labels the molecules adsorbed on the $\mathrm{Fe}_{1}$ and $\mathrm{Fe}_{2}$ centers, respectively. The first dissociation involves $\mathrm{O}_{A}-\mathrm{O}_{B}$. During the dissociation of the second molecule $\mathrm{O}_{A}-\mathrm{O}_{B}$, the distance between atoms $\mathrm{O}_{B}$ and $\mathrm{O}_{b}$ shortens, and a new $\mathrm{O}_{B}-\mathrm{O}_{b}$ molecule is formed from their recombination.

energy path, while the $\mathrm{O}-\mathrm{O}$ bond of the second molecule breaks, a new $\mathrm{O}_{2}$ molecule forms from the recombination of $\mathrm{O}$ atoms adsorbed on the neighboring Fe center (Figure 4a5). This can be clearly seen in Figure $4 b$, which displays the $\mathrm{O}-\mathrm{O}$ distances along the reaction path. The atoms belonging to the two units are labeled $\mathrm{Fe}_{1}-\left(\mathrm{O}_{\mathrm{A}}-\mathrm{O}_{\mathrm{B}}\right)$ and $\mathrm{Fe}_{2}-\left(\mathrm{O}_{\mathrm{a}}-\mathrm{O}_{\mathrm{b}}\right)$. At the beginning of the reaction, both adsorbed molecules have the equilibrium bond length of $1.42 \AA$ (see above). Note, that the Fe atoms relax outward from their initial positions by $\sim 0.8 \AA$ upon $\mathrm{O}_{2}$ adsorption. During the dissociation of the first molecule, the $\mathrm{O}_{\mathrm{A}}-\mathrm{O}_{\mathrm{B}}$ distance increases up to the value of $2.5 \AA$. As soon as the dissociation of the second molecule begins, a clear correlation between the increase of the $\mathrm{O}_{\mathrm{a}}-\mathrm{O}_{b}$ and the decrease of the $\mathrm{O}_{\mathrm{B}}-\mathrm{O}_{\mathrm{a}}$ distances can be seen in Figure $4 \mathrm{~b}$. This shows the formation of a new $\mathrm{O}_{2}$ molecule resulting from the recombination of the preadsorbed molecules. In this reaction intermediate, the $\mathrm{O}_{\mathrm{B}}-\mathrm{O}_{\mathrm{a}}$ bond length equilibrates to the value of $1.38 \AA$. This leads to a metastable $\mathrm{Fe}-\mathrm{O}-\mathrm{O}-\mathrm{Fe}$ bridging configuration that, different from the single $\mathrm{O}_{2}$ bridging case discussed above (Figure 4a2), is now stabilized by the concerted displacement of the metal centers, which is induced by the presence of the $\mathrm{O}$ adsorbates. The recombination is assisted by the high flexibility of the di-iron unit, as shown in Figure 4c: During oxygen dissociation, the $\mathrm{Fe}$ ions move out from the MOCN plane by up to $1.44 \AA$, one at a time and in phase with the corresponding molecular dissociations, while simultaneously reducing their $\mathrm{Fe}-\mathrm{Fe}$ distance to $3.82 \AA$. This distance is sufficiently small to stabilize the bridging configuration. The last step of the reaction is the desorption of the bridging molecular oxygen that leaves an $\mathrm{O}$ atom on each of the metal centers (Figure 3f) and releases a recombined $\mathrm{O}_{b}-\mathrm{O}_{\mathrm{B}}$ molecule (Figure 4a6).
Finally the ligand displacement further lowers the total energy by $1.3 \mathrm{eV}$ (per Fe center) and requires an activation energy of $0.53 \mathrm{eV}$ (Supporting Information, Figure S5). This yields the experimentally observed ligand displacement that is stabilized by both the $\mathrm{O}^{*}$ atoms reaching the supporting metal surface as well as by the formation of a hydrogen bond between the monodentate carboxylate ligand and the phenyl rings of the bisbidentate ligand. During this last reaction step, the organic ligands shift parallel to the surface along the direction of their minor axis, breaking the chelating bidentate bonds with the Fe centers and forming instead a monodentate bond with it. The $\mathrm{O}^{*}$ atom resulting from $\mathrm{O}_{2}$ dissociation (Figure 4a6) moves into the surface region previously occupied by the carboxylate ligand and reaches the $\mathrm{Cu}$ surface. This recovers the four-fold coordination of the Fe centers, which relaxes back toward the surface, decreasing their elevation by $0.5 \AA$.

The reaction mechanism described above competes with a simpler reaction channel in which molecular $\mathrm{O}_{2}$ dissociatively adsorbs on the open regions of the $\mathrm{Cu}(001)$ surface in the MOCN cavities. There are about five hollow sites of the $\mathrm{Cu}(001)$ surface available for $\mathrm{O}_{2}$ dissociation in each cavity (Supporting Information, Figure $\mathrm{S} 1$ ). It is well established that, after the dissociative $\mathrm{O}_{2}$ adsorption, the resulting two $\mathrm{O}$ adatoms occupy two neighboring hollow sites of the surface. ${ }^{40-42}$ According to this mechanism, the $\mathrm{O}$ adatoms could then reach the Fe sites and drive the structural distortion of the MOCN by diffusing underneath the organic overlayer. We have determined the activation energy required to dissociate this $\mathrm{O}$ pair and to bring one adatom close to the nearest Fe center via diffusion below the MOCN (see also Supporting Information). It turns out that the dissociation of the adatom pair requires an activation energy of $0.90 \mathrm{eV}$, in good 
agreement with the recent experimental value of $\sim 1.0 \mathrm{eV} .{ }^{42}$ Even without considering the further diffusion of the $\mathrm{O}$ adatom underneath the MOCN toward the Fe center, this activation energy is already higher than the rate-limiting step of the $\mathrm{O}_{2}$ dissociation on the MOCN di-iron units. In the framework of transition-state theory, this difference would result in a reaction rate for the dissociation on the metal centers that is more than 2 orders of magnitude larger than the one for the reaction mechanism involving the metal surface (usual prefactor of $10^{13} \mathrm{~s}^{-1}$ and room temperature). This proves that besides the experimental evidence obtained from the monoiron structure, the reactivity of the diiron phase is not due to a reaction path involving the $\mathrm{Cu}$ surface.

These results show how the mechanism for $\mathrm{O}_{2}$ dissociation on Fe-based active sites depends on the actual number of metal centers. ${ }^{43}$ The different reactivity displayed by mono- and dinuclear Fe centers toward dioxygen dissociation stems from both structural and electronic effects, namely the presence of the bridging ligands and the ability to assist multielectron processes. ${ }^{44}$ In this context, the reaction mechanism we propose establishes a new element into the structure/function relationship of these active sites: Besides bridging ligation, the reactivity of dinuclear sites is controlled by the structural flexibility of the organic ligands (that allows for their displacement so as to accommodate the $\mathrm{O}^{*}$ atoms) as well as by the possibility of the metal centers to undergo coordinated structural displacements. The dissociation of molecular oxygen is assisted by structural changes in the $\mathrm{Fe}-$ surface and $\mathrm{Fe}-\mathrm{Fe}$ distances by as much as 1.4 and $0.7 \AA$, respectively.

Most of the reaction mechanisms proposed so far for dinuclear $\mathrm{Fe}$ systems involve the presence of bridging $\mathrm{O}_{2}$ across the $\mathrm{Fe}$ centers, ${ }^{43}$ implicitly assuming that the dinuclear site reacts with one $\mathrm{O}_{2}$ molecule. This bridging configuration is present also in our reaction mechanism; however, we show that it originates from two $\mathrm{O}_{2}$ molecules interacting with the dinuclear site. The likelihood of forming such dioxygen bridging species is strongly dependent on the distance between the Fe centers in the dinuclear unit. The large $\mathrm{Fe}-\mathrm{Fe}$ distance in our pristine-phase MOCN system, $4.45 \AA$, is similar to that one of many dinuclear model compounds ${ }^{13,22}$ and prevents the direct formation of such an $\mathrm{O}_{2}$ bridging configuration. This distance is reduced to $3.82 \AA$ (hence allowing the formation of bridging $\mathrm{O}_{2}$ molecules) only during the sequential dissociation of two $\mathrm{O}_{2}$ molecules initially adsorbed side-on on each $\mathrm{Fe}$ center forming the diunit. We notice however that in other di-iron units this distance can display quite noticeable variations, ranging from 2.85 to $5.01 \AA$, ${ }^{45,46}$ and the direct formation of bridging $\mathrm{Fe}_{2}-\mathrm{O}_{2}$ units could be allowed for sufficiently short $\mathrm{Fe}-\mathrm{Fe}$ distances. In addition, the square-planar structure of the surface supported model complex is distinct from the $3 \mathrm{D}$ coordination environment of the synthetic di-iron complexes in which the Fe centers are coordinated in octahedral or square-pyramidal geometries. ${ }^{22}$ Here, the surface enforces the coplanar coordination through the interaction of TPA with the $\mathrm{Cu}$ substrate atoms and spatially confining all the reactants in $2 \mathrm{D}$. The supporting surface might play a role similar to steric hindrance ligands in $3 \mathrm{D}$ compounds and stabilizes the unsaturated four-coordinated structure due to the surfaceimposed adsorption potential. Further, it provides a source of electrons for the reactions. Besides these differences, the key structural resemblance with the carboxylate-bridged dinuclear iron complexes is still remarkable.

In conclusion, our results provide a direct correlation between the reactivity of di-iron active sites and the concerted displacement of metal centers, ligand flexibility, and hydrogenbond formation. Our study reveals the active sites of di-iron MOCNs supported on a metal surface and their interaction with molecular $\mathrm{O}_{2}$. We characterize and identify a novel reaction mechanism that explains the oxygen-induced modifications of MOCNs at the structural and chemical levels. This mechanism displays a cooperative catalytic action of the $\mathrm{Fe}$ ions, is ratelimited by the first $\mathrm{O}_{2}$ dissociation, and exploits the structure of the dinuclear site. Since the di-iron MOCN displays carboxylate ligand shifts analogous to that exhibited by synthetic biomimetic systems ${ }^{22,32,33}$ and biological metalloenzymes sensitive to dioxygen, ${ }^{47,48}$ our characterization and conclusions may be useful for the design of artificial units capable of mimicking the function of enzymes' active sites.

\section{ASSOCIATED CONTENT}

S Supporting Information. Methods, figures of the computational supercells used in the numerical simulations (S1 and S2), simulated STM image for an undissociated $\mathrm{O}_{2}$ molecule as the fourth Fe ligand in the O-modified unit (S3), Löwdin charge population analysis for all stable structures (Table S4), energetics for the ligands shift (S5), and STM image of an additional oxygenated monoiron MOCN (S6). This material is available free of charge via the Internet at http://pubs.acs.org.

\section{AUTHOR INFORMATION}

\section{Corresponding Author}

*E-mail: fabris@democritos.it; phnlin@ust.hk; s.stepanow@ fkf.mpg.de.

\section{Present Addresses}

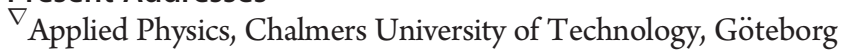
41296, Sweden.

\section{ACKNOWLEDGMENT}

We thank Stephane Pons, Sylvain Clair, and Peter Bencok for their assistance in the XAS experiments at the ESRF in Grenoble. Computational resources from the Computing Centre of the Max-Planck Society in Garching are gratefully acknowledged.

\section{REFERENCES}

(1) Forster, P. M.; Cheetham, A. K. Top. Catal. 2003, 24, 79-86.

(2) Rosseinsky, M. J. Microporous Mesoporous Mater. 2004, 73, 15-30.

(3) Kitagawa, S.; Noro, S.; Nakamura, T. Chem.Commun. 2006, 701-707.

(4) Hocking, R. K.; Brimblecombe, R.; Chang, L.-Y.; Singh, A.; Cheah, M. H.; Glover, C.; Casey, W. H.; Spiccia, L. Nat. Chem. 2011, 3, 461.

(5) Artero, V.; Chavarot-Kerlidou, M.; Fontecave, M. Angew. Chem., Int. Ed. 2011, 50, 7238.

(6) Karunadasa, H. I.; Chang, C. J.; Long, J. R. Nature 2010, 464, 1329.

(7) Holm, R. H.; Kennepohl, P.; Solomon, E. I. Chem. Rev. 1996, 96, 2239.

(8) Collman, J. P.; Wagenknecht, P. S.; Hutchison, J. E. Angew. Chem., Int. Ed. 1994, 33, 1537.

(9) Lippard, S. J. Nat. Chem. Biol. 2006, 2, 504-507.

(10) Sala, X.; Romero, I.; Rodrguez, M.; Escriche, L.; Llobet, A. Angew. Chem., Int. Ed. 2009, 48, 2842.

(11) Que, L. J.; Tolman, W. B. Nature 2008, 455, 333. 
(12) Mirica, L. M.; Vance, M.; Rudd, D. J.; Hedman, B.; Hodgson, K. O.; Solomon, E. I.; Stack, T. D. P Science 2005, 308, 1876.

(13) Wallar, B. J.; Lipscomb, J. D. Chem. Rev. 1996, 96, 2625-2657.

(14) Merkx, M.; et al. Angew. Chem., Int. Ed. 2001, 40, 2782-2807.

(15) Reichard, P. Science 1993, 260, 1773-1777.

(16) Lindqvist, Y.; Huang, Schneider, G.; Shanklin, J. EMBO J. 1996, $15,4081$.

(17) Rosenzweig, A. C.; Frederick, C. A.; Lippard, S. J.; Nordlund, P. Nature 1993, 366, 537-543.

(18) Cadieux, E.; et al. Biochemistry 2002, 41, 10680-10691.

(19) Small, F. J.; Ensign, S. A. J. Biol. Chem. 1997, 272, 24913-24920.

(20) Kovaleva, E. G.; Lipscomb, J. D. Science 2007, 316, 453-457.

(21) Katona, G.; et al. Science 2007, 316, 449-453.

(22) Tshuva, E. Y.; Lippard, S. J. Chem. Rev. 2004, 104, 987-1012.

(23) Dmitriev, A.; Spillmann, H.; Lin, N.; Barth, J. V.; Kern, K. Angew. Chem., Int. Ed. 2003, 42, 2670.

(24) Barth, J. V.; Costantini, G.; Kern, K. Nature 2005, 437, 671679.

(25) Lingenfelder, M. A.; et al. Chem.-Eur. J. 2004, 10, 1913.

(26) Tait, S.; et al. J. Am. Chem. Soc. 2008, 130, 2108-2113.

(27) Stepanow, S.; et al. Nat. Mater. 2004, 3, 229.

(28) Stepanow, S.; Lin, N.; Barth, J. V. J. Phys.: Condens. Matter 2008, 20, 184002 .

(29) Giannozzi, P; et al. J. Phys.: Condens. Matter 2009, 21, 395502; http://www.quantum-espresso.org.

(30) Gambardella, P.; et al. Nat. Mater. 2009, 8, 189-193.

(31) Seitsonen, A. P.; et al. J. Am. Chem. Soc. 2006, 128, 5634-5635.

(32) Lee, D.; Du Bois, J.; Petasis, D.; Hendrich, M. P.; Krebs, C.; Huynh, B. H.; Lippard, S. J. J. Am. Chem. Soc. 1999, 121, 9893-9894.

(33) Lee, D.; Lippard, S. J. J. Am. Chem. Soc. 1998, 120, 12153172154.

(34) Peng, G.; van Elp, J.; Que, L., Jr.; Armstrong, W. H.; Cramer, S. P. J. Am. Chem. Soc. 1995, 117, 2515-2519.

(35) Wasinger, E. C.; de Groot, F. M.; Hedman, B.; Hodgson, K.; Solomon, E. I. J. Am. Chem. Soc. 2003, 125, 12894-12906.

(36) Zheng, F.; Perez-Dieste, V.; McChesney, J. L.; Luk, Y.-Y.; Abbortt, N. L.; Himpsel, F. J. Surf. Sci. 2005, 587, L191-L196.

(37) Note that the Löwdin charges are not an absolute measure of the actual charge localized on the atoms since their value depends on the specific orbitals used to calculate them. On the contrary, their difference is well defined and weakly dependent on the calculation method.

(38) Rovira, C.; Kunc, K.; Hutter, J.; Ballone, P.; Parrinello, M. J. Phys. Chem. A 1997, 101, 8914-8925.

(39) Madsen, G. K. H.; Novák, P. Europhys. Lett. 2005, 69, 777-783.

(40) Hall, J.; Saksager, O.; Chorkendorff, I. Chem. Phys. Lett. 1993, 216, 413.

(41) Yata, M.; Rouch, H. Appl. Phys. Lett. 1999, 75, 1021.

(42) Yagyu, K.; Liu, X.; Yoshimoto, Y.; Nakatsuji, K.; Komori, F. J. Phys. Chem. C 2009, 113, 5541.

(43) Kryatov, S. V.; Rybak-Akimova, E. V. Chem. Rev. 2005, 105, 2175-2226.

(44) Solomon, E. I.; et al. Chem. Rev. 2000, 100, 235-349.

(45) Arii, H.; et al. J. Inorg. Biochem. 2000, 82, 153.

(46) He, C.; Lippard, S. J. Inorg. Chem. 2001, 40, 1414.

(47) Lee, D; Lippard, S. J. Comprehensive Coordination Chemistry II; From Biology to Nanotechnology; Elsevier Ltd.: Oxford, U.K., 2004.

(48) Whittington, D. A.; Lippard, S. J. J. Am. Chem. Soc. 2001, 123, 827. 